\title{
Synergistic Effects of Mixed Solvents on the Solvent Extraction of Copper(II) Benzoates
}

\author{
Hiromichi Yamada*, Kyoko YaJima**, Yujiro NodA* and Hiroko WADA* \\ *Department of Applied Chemistry, Nagoya Institute of Technology, \\ Gokiso, Showa, Nagoya 466, Japan \\ **Nagoya Municipal Industrial Research Institute, \\ Rokuban, Atsuta, Nagoya 456, Japan
}

\begin{abstract}
By using 1-octanol-benzene and 2-octanone-benzene mixed solvents with a wide variety of mixing ratios, the extraction of copper(II) with benzoic acid was carried out at $25^{\circ} \mathrm{C}$ and an aqueous ionic strength of $0.1 \mathrm{~mol} \mathrm{dm}^{-3}\left(\mathrm{NaClO}_{4}\right)$. Each extraction equilibrium for these mixed-solvent systems was determined by a slope analysis and curve-fitting method. The monomeric and dimeric copper(II) benzoates were found to be responsible for all extraction systems. The present results have revealed the relationship between the partition constants of the monomeric extracted species, $\mathrm{CuA}$, and monomeric benzoic acid, HA. It has been proven that the present mixed solvents with a certain composition are superior to chloroform in the extraction of copper(II) by benzoic acid.
\end{abstract}

Keywords Mixed solvent, solvent extraction, solvent effect, copper(II) benzoate, 1-octanol-benzene, 2-octanonebenzene

The selection of a solvent is one of the important subjects in solvent extraction. The most important consideration in the selection of a solvent is the extractability of the concerned elements. From this point, chloroform has frequently been used as the most useful solvent in solvent extraction. Recently, the preservation of the natural environment has been widely advocated. This problem should be considered when selecting a solvent for an investigation on solvent extraction. From this point of view, halogenated solvents, such as chloroform, 1,2-dichloroethane and carbon tetrachloride, should be controlled regarding their use as much as possible. It is thus urgently necessary to develop a nature-friendly and substituteuseful solvent for these halogenated solvents.

In a previous investigation ${ }^{1}$ of solvent effects on the extraction of copper(II) with benzoic acid, a pronounced difference in the extraction behavior between benzene and chloroform was found; that is, in the extraction of copper(II) benzoates using benzene as a solvent the emulsion appeared in the region where the percent extraction of copper exceeded about $20 \%$; however, in the region where the emulsion is not formed, extraction using benzene as a solvent has turned out to begin at a lower $\mathrm{pH}$ region than that using chloroform. It was presumed that the superiority of chloroform over benzene, 1-octanol and 2-octanone can be attributed to the low polarity, moderate solvating ability and low solubility of water in it. The emergence of the emulsion in the extraction of copper(II) benzoates using benzene can be expected to arise mainly from the lack of solvating ability in benzene. Therefore, using mixed solvents prepared by the addition of 1-octanol or 2-octanone, which has a solvating ability, to benzene, the extraction of copper(II) benzoates can be expected to result in the disappearance of the emulsion as well as high extractability.

In the present work the extraction equilibrium of copper(II) with benzoic acid using these mixed solvents with various compositions was analyzed by slope analysis and curve-fitting methods; ${ }^{1,2}$ mixed solvents ranging in certain limited compositions have been found to be superior to chloroform in the extraction of copper(II) with benzoic acid. In addition, the solvent effect on the relationship between the partition constants of the monomeric copper(II) benzoate $\mathrm{CuA}_{2}$ and monomeric benzoic acid has been satisfactorily interpreted.

\section{Experimental}

\section{Reagents}

Mixed solvents were prepared in the same manner as that described previously. ${ }^{3}$ Copper(II) perchlorate, benzoic acid and the other reagents were of reagent grade and used without further purification. Aqueous solutions were prepared with distilled deionized water.

\section{Procedure}

The extraction of copper(II) by benzoic acid using eleven kinds of 1-octanol-benzene and 2-octanonebenzene mixed solvents (listed in Tables 1 and 2) was 
Table 1 Extraction constants in the 1-octanol-benzene mixed-solvent systems

\begin{tabular}{|c|c|c|c|c|c|c|c|}
\hline \multirow{2}{*}{$\frac{\text { No. }}{00}$} & \multicolumn{2}{|c|}{$C_{\mathrm{ROH}}$} & \multirow{2}{*}{$\frac{\log K_{\mathrm{ex}(10)}}{-6.65}$} & \multirow{2}{*}{$\frac{\log K_{\operatorname{ex}(11)}}{-}$} & \multirow{2}{*}{$\frac{\log K_{\mathrm{ex}(20)}}{-}$} & \multirow{2}{*}{$\frac{\log K_{\mathrm{ex}(21)}}{-}$} & \multirow{2}{*}{$\frac{\log K_{\mathrm{ex}(22)}}{-6.89}$} \\
\hline & 0 & $(0)$ & & & & & \\
\hline 0 & $\mathbf{0}$ & $(0)$ & - & -4.40 & - & - & -4.12 \\
\hline 1 & 0.1 & $(1.5)$ & -7.20 & -6.11 & -10.27 & - & -7.99 \\
\hline 2 & 0.25 & ( 3.7$)$ & -7.28 & - & -11.20 & -9.83 & - \\
\hline 3 & 0.3 & ( 4.5$)$ & -7.51 & - & -11.13 & -10.75 & - \\
\hline 4 & 0.4 & $(6.0)$ & -7.68 & - & -11.81 & -11.39 & - \\
\hline 5 & 0.5 & ( 7.5$)$ & -7.68 & - & -11.58 & - & - \\
\hline 6 & 1.0 & ( 15.1) & -7.99 & - & -12.16 & - & - \\
\hline 7 & 2.0 & ( 30.5$)$ & -8.03 & - & -12.64 & - & - \\
\hline 8 & 3.0 & ( 46.5) & -8.27 & - & -13.39 & - & - \\
\hline 9 & 4.0 & ( 62.5$)$ & -8.15 & - & -13.46 & - & - \\
\hline 10 & 5.0 & $(78.6)$ & -8.09 & - & -13.54 & - & - \\
\hline 11 & 6.0 & (94.6) & -7.82 & - & -13.36 & - & - \\
\hline 12 & 6.34 & $(100)$ & -7.81 & - & -13.34 & - & - \\
\hline
\end{tabular}

$C_{\mathrm{ROH}}$ denotes the molar concentration of 1-octanol in the mixed solvents and the values in parentheses denote the concentration of percentage by volume. No. 00, 0 and 12 denote chloroform, benzene and 1-octanol, respectively.

carried out in a thermostatic bath kept at $25 \pm 0.2^{\circ} \mathrm{C}$. The ionic strength of the aqueous phase was adjusted to $0.1 \mathrm{~mol} \mathrm{dm}^{-3}$ using sodium perchlorate. The total concentration of copper(II) was $5 \times 10^{-3} \mathrm{~mol} \mathrm{dm}^{-3}$, and that of benzoic acid was in the region of $0.1-0.5 \mathrm{~mol} \mathrm{dm}^{-3}$ for 1 octanol-benzene mixed solvents and $0.2-0.5 \mathrm{~mol} \mathrm{dm}^{-3}$ for 2-octanone-benzene ones, respectively. The other procedures for the extraction of copper(II) with benzoic acid were the same as those used in previous work. ${ }^{1}$

\section{Apparatus}

For mechanical shaking, centrifugation and a determination of the hydrogen-ion concentration in the aqueous phase the same apparatus as those in the previous work were used. ${ }^{1}$

\section{Results and Discussion}

Analysis of the extraction equilibrium of copper(II) with benzoic acid in each mixed-solvent system

From the previous results ${ }^{1}$ concerning the extraction of copper(II) with benzoic acid using benzene, 1-octanol, and 2-octanone as a diluent, copper(II) can be expected to be extracted as the monomeric and dimeric benzoates in the present mixed-solvent systems. Thus, the following expression can be derived in a similar manner as previously described: ${ }^{1}$

$$
\begin{aligned}
& \log C_{\mathrm{Cu}, 0}-\left(\log \left[\mathrm{Cu}^{2+}\right]-2 \log \left[\mathrm{H}^{+}\right]\right)-\log K_{\mathrm{ex}(1 a)} \\
& -(2+a) \log [\mathrm{HA}]_{\text {。 }}
\end{aligned}
$$



where $K_{\mathrm{ex}(1 a)}$ and $K_{\mathrm{ex}(2 b)}$ denote the extraction constants of the monomeric and dimeric copper(II) benzoates
$\left(\mathrm{CuA}_{2}(\mathrm{HA})_{a}\right.$ and $\left.\mathrm{Cu}_{2} \mathrm{~A}_{4}(\mathrm{HA})_{b}\right)$, respectively. Irrespective of the composition of a mixed solvent, the monomeric and dimeric copper(II) benzoates have been found to be responsible for the extraction of copper(II) with benzoic acid, since the plots of $\log C_{\mathrm{Cu}, \mathrm{O}}-\log \left[\mathrm{Cu}^{2+}\right]+$ $2 \log \left[\mathrm{H}^{+}\right]$against $\log \left[\mathrm{Cu}^{2+}\right]-2 \log \left[\mathrm{H}^{+}\right]$at constant $[\mathrm{HA}]_{\mathrm{o}}$ based on Eq. (1) fit well to the normalized curve, $\log (1+X) v s . \log X$, in the each mixed solvent system for both the 1-octanol-benzene and 2-octanone-benzene systems. The concentrations of $\mathrm{Cu}^{2+}$ were calculated from the relations of $\left[\mathrm{Cu}^{2+}\right]=C_{\mathrm{Cu}, w} / \alpha_{\mathrm{Cu}(\mathrm{A})}$ and $\alpha_{\mathrm{Cu}(\mathrm{A})}=1+$ $K_{1}\left[\mathrm{~A}^{-}\right]+K_{1} K_{2}\left[\mathrm{~A}^{-}\right]^{2}$ under the respective conditions, where $\left[\mathrm{A}^{-}\right]$was calculated using $\left[\mathrm{A}^{-}\right]=C_{\mathrm{HA}} /\left\{1+\left(\left[\mathrm{H}^{+}\right] /\right.\right.$ $\left.K_{\mathrm{a})}\right){ }^{1}$

As previously noted ${ }^{1}$, the following equations are available for the determination of the number of benzoic acid involved in the monomeric and dimeric copper(II) species, respectively:

$$
\begin{gathered}
\log C_{\mathrm{Cu}, \mathrm{o}}-\left(\log \left[\mathrm{Cu}^{2+}\right]-2 \log \left[\mathrm{H}^{+}\right]\right) \\
=\log \left(\sum_{a} K_{\mathrm{ex}(1 a)}[\mathrm{HA}]_{\left.\mathrm{o}^{(2+a)}\right)}\right.
\end{gathered}
$$

and

$$
\begin{gathered}
\log C_{\mathrm{Cu}, \mathrm{o}}-2\left(\log \left[\mathrm{Cu}^{2+}\right]-2 \log \left[\mathrm{H}^{+}\right]\right) \\
=\log \left(2 \sum_{b} K_{\mathrm{ex}(2 b)}[\mathrm{HA}]_{\left.\mathrm{o}^{(4+b)}\right)} .\right.
\end{gathered}
$$

Concerning the monomeric extracted species plots of $\left\{\log C_{\mathrm{Cu}, o}-\left(\log \left[\mathrm{Cu}^{2+}\right]-2 \log \left[\mathrm{H}^{+}\right]\right)\right\}$against $\log [\mathrm{HA}]_{\mathrm{o}}$ fitting well with a straight line with a slope of 2.0 in the 1-octanol-benzene mixed solvent systems, except for system No. 1, only $\mathrm{CuA}_{2}$ has been found to be responsible for the extraction, and the respective extraction constants were determined from the intercept of each straight line with a slope of 2.0 (Table 1). In the 2-octanonebenzene mixed solvents, the plots fitted well with a 
Table 2 Extraction constants in the 2-octanone-benzene mixed-solvent systems

\begin{tabular}{cccccccc}
\hline No. & \multicolumn{2}{c}{$C_{\mathrm{RCO}}$} & $\log K_{\mathrm{ex}(10)}$ & $\log K_{\operatorname{ex}(11)}$ & $\log K_{\mathrm{ex}(20)}$ & $\log K_{\operatorname{ex}(21)}$ & $\log K_{\mathrm{ex}(22)}$ \\
\hline 13 & 0.1 & $(1.5)$ & - & -5.37 & -9.47 & - & -6.25 \\
14 & $0.2(3.0)$ & - & -5.22 & -9.68 & - & -6.66 \\
15 & $0.3(4.4)$ & -7.25 & -5.99 & -9.70 & - & -7.10 \\
16 & $0.4(6.0)$ & -7.16 & - & -10.10 & - & -7.34 \\
17 & $0.5(7.5)$ & -7.27 & - & -10.25 & - & -7.99 \\
18 & $1.0(15.0)$ & -8.07 & - & -11.74 & -10.22 & - \\
19 & $2.0(30.1)$ & -8.36 & - & -11.67 & -11.11 & - \\
20 & $3.0(45.6)$ & -8.46 & - & -12.00 & - & - \\
21 & $4.0(61.5)$ & -8.48 & - & -12.51 & - & - \\
22 & $5.0(77.6)$ & -8.48 & - & -12.94 & - & - \\
23 & $6.0(94.3)$ & -8.52 & - & -13.02 & - & - \\
24 & $6.40(100)$ & -8.53 & - & -13.28 & - & - \\
\hline
\end{tabular}

$C_{\mathrm{RCO}}$ denotes the molar concentration of 2-octanone in the mixed solvents and the values in parentheses denote the concentration of percentage by volume. No. 24, refers to 2 -octanone.

straight line with a slope of 2.0 for No. 16 - 23, and 3.0 for No. 13 and 14 , respectively. The respective extraction constants of $\mathrm{CuA}_{2}$ and $\mathrm{CuA}_{2} \mathrm{HA}$ were estimated based on the intercept of each straight line (Table 2). In the mixed-solvent systems of No. 1 (1-octanol-benzene mixed solvent) and No. 15 (2-octanone-benzene mixed solvent) the plots deviated upward from a straight line with a slope of 2.0 with increasing [HA] . This suggests that two kinds of monomeric copper(II) species are responsible for extraction in these mixed-solvent systems, and that one of them might be $\mathrm{CuA}_{2}$. Thus, the following expression can be derived from Eq. (2):

$$
\begin{gathered}
\log C_{\mathrm{Cu}, \mathrm{o}}-\left(\log \left[\mathrm{Cu}^{2+}\right]-2 \log \left[\mathrm{H}^{+}\right]\right)-2 \log [\mathrm{HA}]_{\mathrm{o}} \\
=\log K_{\mathrm{ex}(10)}+\log \left(1+\frac{K_{\text {ex(1a) }}}{K_{\mathrm{ex}(10)}}[\mathrm{HA}]_{\mathrm{o}}{ }^{a}\right) .
\end{gathered}
$$

According to a similar curve-fitting method to that employed previously ${ }^{4}$, as plots of the values of the lefthand side of the above equation against $\log [\mathrm{HA}]_{\mathrm{o}}$ fall on the normalized curve, $\log (1+X)$ vs. $\log X$, the other monomeric species was found to be $\mathrm{CuA}_{2} \mathrm{HA}$, and each extraction constant was estimated, as in Tables 1 and 2.

On the other hand, concerning the dimeric extracted species, the plots of $\left\{\log C_{\mathrm{Cu}, 0}-2\left(\log \left[\mathrm{Cu}^{2+}\right]-2 \log \left[\mathrm{H}^{+}\right]\right)\right\}$ against $\log [\mathrm{HA}]_{\mathrm{o}}$ fall on a straight line with a slope of 4.0; the dimeric copper(II) species was found to be $\mathrm{Cu}_{2} \mathrm{~A}_{4}$, and the each extraction constant was estimated from the intercept of the each straight line for the following mixedsolvent systems: No. 5-11, and No. 20-23. In the other mixed-solvent systems the plots of $\left\{\log C_{\mathrm{Cu}, \mathrm{o}}-\right.$ $\left.2\left(\log \left[\mathrm{Cu}^{2+}\right]-2 \log \left[\mathrm{H}^{+}\right]\right)\right\}$against $\log [\mathrm{HA}]_{0}$ deviated upward from a straight line with a slope of 4.0 with increasing [HA $]_{0}$. This tendency suggests that $\mathrm{Cu}_{2} \mathrm{~A}_{4}$ and $\mathrm{Cu}_{2} \mathrm{~A}_{4}(\mathrm{HA})_{b}(b \geqq 1)$ can be expected to be responsible for the extraction. Thus, the following equation can be obtained from Eq. (3):

$$
\begin{gathered}
\log C_{\mathrm{Cu}, \mathrm{o}}-2\left(\log \left[\mathrm{Cu}^{2+}\right]-2 \log \left[\mathrm{H}^{+}\right]\right)-4 \log [\mathrm{HA}]_{o} \\
=\log 2+\log K_{\mathrm{ex}(20)}+\log \left(1+\frac{K_{\mathrm{ex}(2 b)}}{K_{\mathrm{ex}(20)}}[\mathrm{HA}]_{o}^{b}\right) .
\end{gathered}
$$

By a similar curve-fitting method as that for analyzing the monomeric species, the values of the left-hand side of Eq. (5) were plotted against $\log [\mathrm{HA}]_{\text {o. }}$. The plots were fitted with the normalized curve, $\log \left(1+X^{2}\right) v s . \log X$, in the following mixed-solvent systems: No. 1 and No. $13-$ 17; also, the dimeric species were proven to be $\mathrm{Cu}_{2} \mathrm{~A}_{4}$ and $\mathrm{Cu}_{2} \mathrm{~A}_{4}(\mathrm{HA})_{2}$ in these mixed-solvent systems. For systems No. 2-4, 18 and 19, the plots fall on the normalized curve, $\log (1+X)$ vs. $\log X$. The dimeric species, $\mathrm{Cu}_{2} \mathrm{~A}_{4}$ and $\mathrm{Cu}_{2} \mathrm{~A}_{4} \mathrm{HA}$, were found to be extracted in these mixed-solvent systems. Each extraction constant was estimated as previously employed. ${ }^{4}$ The values are summarized in Tables 1 and 2 together with the previous results $^{1}$ for chloroform, benzene, 1-octanol and 2octanone.

\section{Estimation of the partition constants of $\mathrm{CuA}_{2}$}

The monomeric copper(II) benzoate $\mathrm{CuA}_{2}$, has been found to be responsible for extraction in the present mixed-solvent systems other than systems No. 13 and 14 . The extraction constant of the species can be written as

$$
K_{\mathrm{ex}(10)}=\left[\mathrm{CuA}_{2}\right]_{0}\left[\mathrm{H}^{+}\right]^{2} /\left[\mathrm{Cu}^{2+}\right][\mathrm{HA}]_{0^{2}}{ }^{2},
$$

and can be rewritten as follows using the four kinds of equilibrium constants:

$$
K_{\text {ex }(10)}=K_{\mathrm{D}, \mathrm{CuA} 2} K_{\mathrm{D}, \mathrm{HA}}{ }^{-2} \beta_{\mathrm{CuA} 2} K_{\mathrm{a}}{ }^{2},
$$

where $K_{\mathrm{D}, \mathrm{CuA} 2}, K_{\mathrm{D}, \mathrm{HA}}, \beta_{\mathrm{CuA} 2}$ and $K_{\mathrm{a}}$ represent the partition constant of $\mathrm{CuA}_{2}$, that of the monomeric benzoic acid, the overall formation constant of $\mathrm{CuA}_{2}$ in the aqueous phase, and the dissociation constant of benzoic acid in the aqueous phase, respectively. The partition constant 
Table 3 The partition and dimerization constants of monomeric benzoic acid and monomeric copper(II) benzoate $\mathrm{CuA}$

\begin{tabular}{|c|c|c|c|c|c|c|c|c|c|}
\hline No. & $\log K_{\mathrm{D}, \mathrm{HA}}$ & $\log K_{2 . \mathrm{HA}}$ & $\log K_{\mathrm{D}, \mathrm{CuA} 2}$ & $\log K_{2, \mathrm{CuA} 2}$ & No. & $\log K_{\mathrm{D} . \mathrm{HA}}$ & $\log K_{2, \mathrm{HA}}$ & $\log K_{\mathrm{D}, \mathrm{CuA} 2}$ & $\log K_{2, \mathrm{CuA} 2}$ \\
\hline 00 & 0.76 & 1.46 & - & - & & & & & \\
\hline 0 & 0.14 & 2.42 & - & - & & & & & \\
\hline 1 & 0.81 & 1.05 & 0.72 & 4.13 & 13 & 0.53 & 1.63 & - & - \\
\hline 2 & 1.07 & 0.52 & 1.16 & 3.36 & 14 & 0.59 & 1.52 & - & - \\
\hline 3 & 1.21 & 0.16 & $1.2 \mathrm{I}$ & 3.89 & 15 & 0.67 & 1.37 & 0.39 & 4.80 \\
\hline 4 & 1.31 & -0.09 & 1.24 & 3.55 & 16 & 0.74 & 1.23 & 0.62 & 4.22 \\
\hline 5 & 1.39 & -0.43 & 1.40 & 3.78 & 17 & 0.85 & 1.02 & 0.73 & 4.29 \\
\hline 6 & 1.63 & - & 1.57 & 3.82 & 18 & 1.18 & 0.31 & 0.59 & 4.40 \\
\hline 7 & 1.75 & - & 1.77 & 3.42 & 19 & 1.33 & 0.03 & 0.60 & 5.05 \\
\hline 8 & 1.84 & - & 1.71 & 3.15 & 20 & 1.50 & -0.41 & 0.84 & 4.92 \\
\hline 9 & 1.89 & - & 1.93 & 2.84 & 21 & 1.64 & -0.86 & 1.10 & 4.45 \\
\hline 10 & 1.88 & - & 1.97 & 2.64 & 22 & 1.78 & - & 1.38 & 4.02 \\
\hline 11 & 1.86 & - & 2.20 & 2.28 & 23 & 1.85 & - & 1.48 & 4.02 \\
\hline 12 & 1.84 & - & 2.17 & 2.28 & 24 & 1.91 & - & 1.59 & 3.78 \\
\hline
\end{tabular}

The values of $K_{\mathrm{D} . \mathrm{HA}}$ and $K_{2, \mathrm{HA}}$ were cited from the previous work. ${ }^{3} \quad$ Numbers are the same as in Tables 1 and 2.

of the monomeric benzoic acid was estimated for the present mixed-solvent systems in a previous investigation. ${ }^{3}$ The values are summarized in Table 3 together with the other constants. The overall formation constant of $\mathrm{CuA}_{2}$ in the aqueous phase was calculated from the stepwise formation constants $\left(K_{1}\right.$ and $\left.K_{2}\right)$ determined in a previous study. ${ }^{1}$ The dissociation constant of benzoic acid in the aqueous phase is known to be $\mathrm{p} K_{\mathrm{a}}=4.04$ at $25^{\circ} \mathrm{C}$ and in $0.1 \mathrm{~mol} \mathrm{dm}^{-3}$ perchlorate medium in the previous work. ${ }^{5}$ The extraction constant of $\mathrm{CuA}_{2}$ was estimated (Tables 1 and 2) in the present investigation. Thus, the value of the partition constant of $\mathrm{CuA}_{2}$ can be calculated using Eq. (7). The values are summarized in Table 3 together with the partition constants of monomeric benzoic acid estimated previously. ${ }^{3}$

\section{Relationship between the partition constants of $\mathrm{CuA}_{2}$ and} monomeric benzoic acid

In our previous studies ${ }^{4,6}$ concerning the extraction of copper(II) with decanoic acid using some alcohols and ketones as a solvent, the dimerization of copper(II) decanoate in the organic phase was successfully explained by means of the regular solution theory, which proven to be available for interpretating the solvent effects on solvent extraction among a homologous series of solvents differing in their chain length, even for a solvating solvent. From the additive nature of the molar cohesive energy and molar volume of the ligand in a complex, the partition constant of a complex $\mathrm{MA}_{n}$ can be approximately correlated to that of the ligand (HA) as: $K_{\mathrm{D} . \mathrm{MA} n} \simeq K_{\mathrm{D} . \mathrm{HA}}{ }^{n}{ }^{7}$ As can be seen from Table 3, this quantitative relationship between the partition constants of $\mathrm{CuA}_{2}$ and $\mathrm{HA}$ does not hold for the present extraction systems. The partition constant of $\mathrm{CuA}_{2}$ for each mixed solvent system is significantly smaller than that speculated by $K_{\mathrm{D}, \mathrm{CuA} 2}=K_{\mathrm{D}, \mathrm{HA} .}{ }^{2} \quad$ This is attributable to the solvation and/or hydration of $\mathrm{CuA}_{2}$ and the HA species. If the interaction between these species and the solvent can be neglected, that is $K_{\mathrm{D}, \mathrm{CuA} 2}=K_{\mathrm{D}, \mathrm{HA}}{ }^{2}$, the extraction constant of $\mathrm{CuA}_{2}\left(K_{\mathrm{ex}(10)}\right)$ is equal to $\beta_{\mathrm{CuA} 2} K_{\mathrm{a}}^{2}$ in the present extraction systems irrespective of the organic solvent, or $\log K_{\text {ex }(10)}=\log \beta_{\mathrm{CuA} 2}+2 \log K_{\mathrm{a}}=-6.30$, which may be called the "theoretical extraction constant". In all mixed-solvent systems, the extraction constant of $\mathrm{CuA}_{2}$ is considerably smaller than this value, suggesting that the monomeric benzoic acid is more strongly affected by the solvent than the monomeric copper(II) benzoate $\mathrm{CuA}_{2}$. Thus, the conditional partition constant of HA can be regarded as becoming greater by solvation, as mentioned in a previous study. ${ }^{1}$

The relationship between the partition constants of the monomeric copper(II) species $\mathrm{CuA}_{2}$ and the monomeric benzoic acid is shown in Fig. 1. The plots fall on three kinds of straight lines with a slope of 2.0. These straight lines with a different intercept correspond to a similar series of solvents to benzene, to 1-octanol and to 2octanone from left to right, successively. The plots for the 1-octanol-benzene mixed-solvent systems vary widely. This is attributable to the increase in the solubility of water in mixed solvents with increasing the concentration of 1-octanol in the mixed solvents, that is, the increase in the degree of hydration of benzoic acid in the mixed solvents. As mentioned concerning a previous investigation ${ }^{3}$, this is also supported by decreasing the conditional partition constant of the monomeric benzoic acid with increasing the concentration of 1octanol in the 1-octanol-benzene mixed solvents, in contrast to the 2-octanone-benzene mixed solvents. Thus, the straight line is drawn so that the plots of No. 11 and 12 are shifted to the left from it.

The following relation between the partition constants of $\mathrm{CuA}_{2}$ and HA can hold: $K_{\mathrm{D}, \mathrm{CuA} 2}=C K_{\mathrm{D}, \mathrm{HA}}{ }^{2}$, where $C$ is a certain constant corresponding to each similar series of solvents. If $K_{\mathrm{D}, \mathrm{CuA} 2}=K_{\mathrm{D}, \mathrm{HA}}{ }^{2}$, the plots should fall on a straight line with a slope of 2.0 drawn through the origin, which is shown by the dotted line in Fig. 1. The plot for the chloroform system lies close to this dotted straight line. The extraction constant of $\mathrm{CuA}_{2}$ in the chloro- 


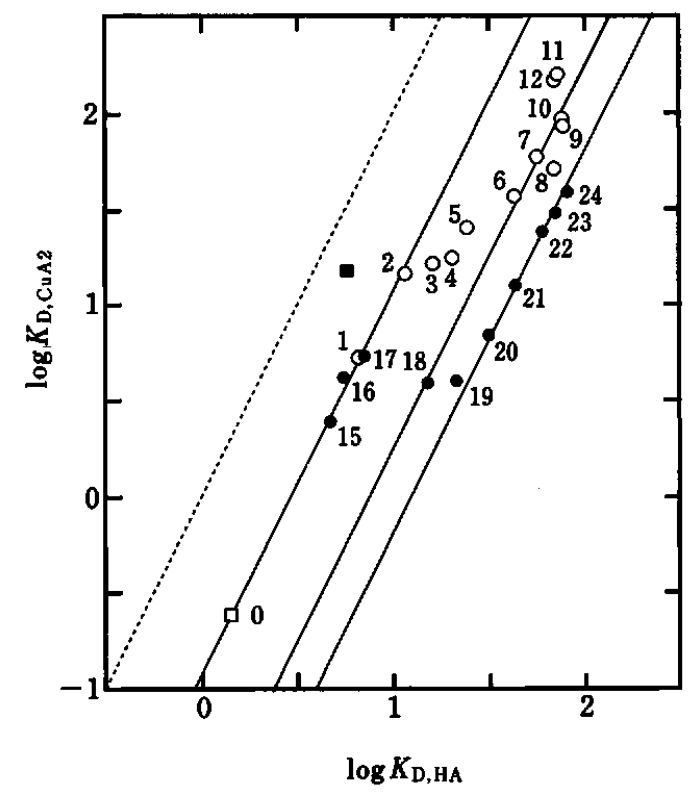

Fig. 1 Relationship between the partition constants of the monomeric copper(II) benzoate $\mathrm{CuA}_{2}$ and the monomeric benzoic acid. The solid and dotted lines are straight lines with a slope of 2.0 , respectively. The numbers refer to the solvents shown in Tables 1 and 2 . The solid and open squares refer to chloroform and benzene, respectively.

form system, which was estimated in the previous investigation ${ }^{1}$, should be $\log K_{\text {ex }(10)}=-6.65$, and is close to the "theoretical extraction constant" noted above. This suggests that chloroform is a good solvent for the extraction of monomeric copper(II) benzoate, $\mathrm{CuA}_{2}$.

On the other hand, in the extraction of copper(II) with benzoic acid using benzene as a solvent, the $\mathrm{CuA}_{2}$ species was not observed to any appreciable extent. As shown in Table 1, copper(II) is extracted as $\mathrm{CuA}_{2} \mathrm{HA}$ and $\mathrm{Cu}_{2} \mathrm{~A}_{4}(\mathrm{HA})_{2}$. The partition constant of $\mathrm{CuA}_{2}$ should fall on a line corresponding to a similar series of solvents to benzene in Fig. 1. From the value of the partition constant of $\mathrm{HA}$, it can be inferred to be $\log K_{\mathrm{D}, \mathrm{CuA} 2}=$ -0.62 , as shown by the open square symbol in Fig. 1 .

This low value can be anticipated to result in the appearance of the emulsion in this system. This is attributable primarily to the hydration of benzoic acid in the benzene phase, as reported by Fujii et al. ${ }^{8}$ In addition, from the value of $K_{\mathrm{D}, \mathrm{CuA} 2}$ the extraction constant of $\mathrm{CuA}_{2}$, which was not observed in the benzene system, was calculated using Eq. (7) to be $\log K_{\text {ex(10) }}=$ -7.20 . In one of a series of investigations concerning the solvent effects on the extraction of some metals by various $\beta$-diketones, Suzuki et al. ${ }^{9}$ explained by the regular solution theory that irrespective of the solvent used, except for methylene chloride-chlorobenzene mixed solvents and methylene chloride, the plots of $\log D_{\mathrm{M}}$ ( $D_{\mathrm{M}}$ : the distribution ratio of scandium thenoyltrifluoroacetonate at $10^{-7.8} \mathrm{~mol} \mathrm{dm}^{-3}$ thenoyltrifluoroacetonate ion) against $\log D_{\beta}\left(D_{\beta}\right.$ : the distribution coefficient of thenoyltrifluoroacetone) fell on the same straight line with a slope of about 3 in the extraction systems using carbon tetrachloride, benzene, $o$-dichlorobenzene, chlorobenzene, ethyl bromide, methylene chloride, carbon tetrachloride-ethyl bromide, benzene-ethyl bromide, benzene-o-dichlorobenzene and methylene chloridechlorobenzene mixed solvents. The difference between their and our results can be ascribed to the difference in the solvating ability of the solvents used by them and us. In other words, since their solvents are regarded as nonsolvating or low-solvating solvents, thenoyltrifluoroacetone and its scandium chelate are anticipated to interact with all solvent molecules in a similar way. In the present systems benzene is a nonsolvating solvent, and 1-octanol and 2-octanone are solvating solvents which differ from one another in their solvating ability, as mentioned in a previous study ${ }^{3}$ concerning the solvation constant of monomeric benzoic acid with each solvent molecule. Therefore, since $\mathrm{CuA}_{2}$ and HA interact with each solvent molecule to a different extent, the plots fall on the respective straight lines with a different intercept.

Relationship between the dimerization and partition constants of $\mathrm{CuA}_{2}$

According to the results obtained by the present study, the following dimerization of copper(II) benzoate in mixed solvents can be present, except for mixed solvents No. 13 and 14:

$$
2\left(\mathrm{CuA}_{2}\right)_{\mathrm{o}} \rightleftharpoons\left(\mathrm{Cu}_{2} \mathrm{~A}_{4}\right)_{\mathrm{o}}
$$

with the dimerization constant $K_{2, \mathrm{CuA} 2}$, which can be calculated from the respective extraction constants as follows: $K_{2, \mathrm{CuA} 2}=K_{\mathrm{ex}(20)} / K_{\mathrm{ex}(10)^{2}}$. The values estimated using this equation are summarized in Table 3. Furthermore, the dimerization of the monomeric copper(II) benzoate $\mathrm{CuA}_{2}$ can be represented by the following scheme:

$$
\begin{gathered}
2\left(\mathrm{CuA}_{2}\right)_{\mathrm{o}} \stackrel{K_{2, \mathrm{CuA} 2}}{\rightleftharpoons}\left(\mathrm{Cu}_{2} \mathrm{~A}_{4}\right)_{\mathrm{o}} \\
\frac{\text { organic phase }}{\text { aqueous phase }} \| K_{\mathrm{D}, \mathrm{CuA} 2} \\
2\left(\mathrm{CuA}_{2}\right)_{\mathrm{w}} \stackrel{K_{2, \mathrm{CuA} 2(\mathrm{w})}}{\rightleftharpoons}\left(\mathrm{Cu}_{2} \mathrm{~A}_{4}\right)_{\mathrm{w}, \mathrm{w} 2 \mathrm{~A} 4},
\end{gathered}
$$

where $K_{2, \mathrm{CuA} 2(w)}$ and $K_{\mathrm{D}, \mathrm{Cu} 2 \mathrm{~A} 4}$ denote the dimerization constant of $\mathrm{CuA}_{2}$ in the aqueous phase and the partition constant of the dimeric copper(II) benzoate $\mathrm{Cu}_{2} \mathrm{~A}_{4}$, respectively. Since the free-energy change is independent of the reaction process, the following relationship among the four kinds of equilibrium constants described above can apply in the above scheme:

$$
2 \log K_{\mathrm{D}, \mathrm{CuA} 2}+\log K_{2, \mathrm{CuA} 2}=\log K_{2, \mathrm{CuA} 2(\mathrm{w})}+\log K_{\mathrm{D}, \mathrm{Cu} 2 \mathrm{~A} 4 .}
$$

The dimerization constant of $\mathrm{CuA}_{2}$ in the aqueous phase $\left(K_{2, \mathrm{CuAz}(w)}\right)$ can be regarded as being constant irrespective of the composition of the organic phase. In addition, it 


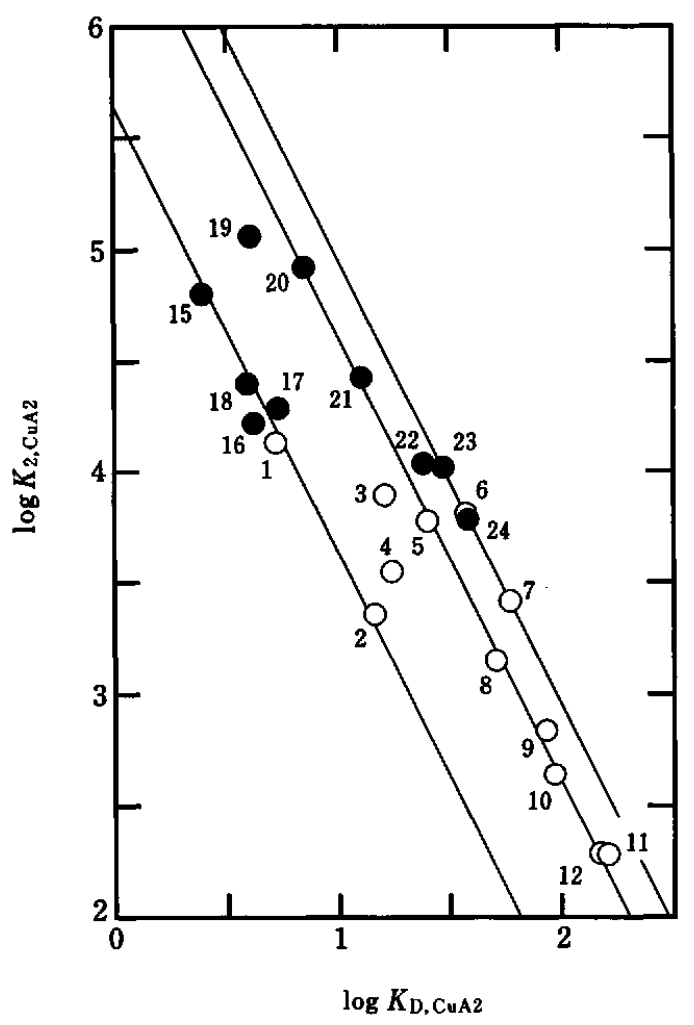

Fig. 2 Relationship between the dimerization and partition constants of the monomeric copper(II) benzoate $\mathrm{CuA}_{2}$. The numbers are the same as in Fig. 1. The solid lines are straight lines with a slope of -2.0 .

can be expected that there is no pronounced difference in the value of the partition constant of $\mathrm{Cu}_{2} \mathrm{~A}_{4}$ among a homologous series of solvents, since the dimeric species $\left(\mathrm{Cu}_{2} \mathrm{~A}_{4}\right)$ may be hardly influenced by the solvent compared with the monomeric species, $\mathrm{CuA}_{2}$. Then, the value of the right-hand side of Eq. (8) can be approximated to be constant. Accordingly, the plots of $\log K_{2, \mathrm{CuA} 2}$ against $\log K_{\mathrm{D}, \mathrm{CuA} 2}$ can be expected to lie on a straight line with a slope of -2.0 . As expected, the plots shown in Fig. 2 fall on three kinds of straight lines with a slope of -2.0 , which correspond to similar solvents to benzene, to 1-octanol and to 2-octanone from left to right in a similar manner as the results in Fig. 1. In contrast with the results in Fig. 1, the plots on the 2-octanonebenzene mixed solvents display no good linear relationship, except for the mixed solvents containing a small amount of 2-octanone. This can be accounted for as follows. Though the partition of $\mathrm{CuA}_{2}$ is affected by the solvent to a similar extent as that of monomeric benzoic acid in 2-octanone-benzene mixed solvents containing more than $2 \mathrm{~mol} \mathrm{dm}^{-3}$ of 2-octanone (No. 19 23), and pure 2-octanone (No. 24), the mixed solvents ranging in concentration of 2-octanone from 2 to $5 \mathrm{~mol}$ $\mathrm{dm}^{-3}$ (No. 19-22) among these mixed solvents can be expected to act as a different solvent belonging to neither a similar series of solvents to 2-octanone nor those to benzene for the dimerization of $\mathrm{CuA}_{2}$. That is to say,

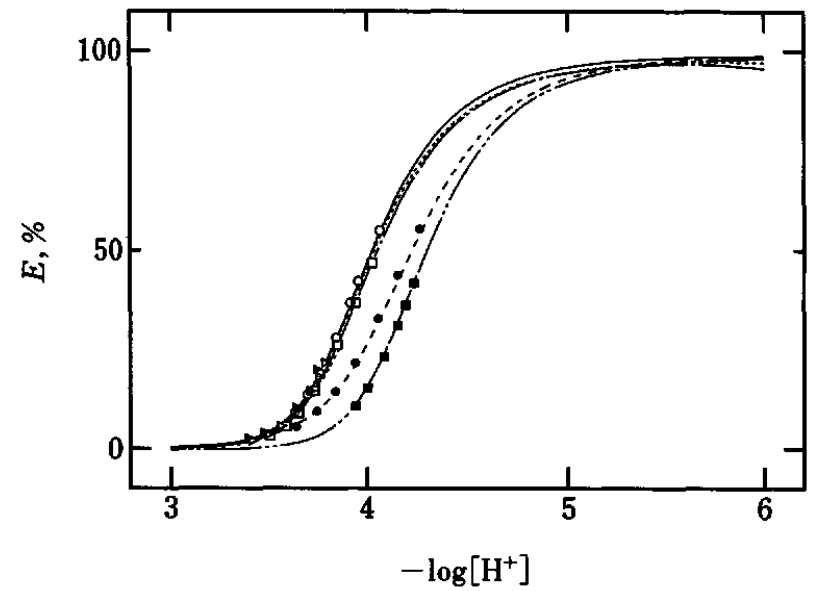

Fig. 3 Comparison of mixed solvents with benzene, 1octanol, 2-octanone and chloroform. The symbols and lines are as follows: $O$ and - , mixed solvent No. 6; $\square$ and -----, mixed solvent No. 18; $\Delta$ and -.-.-, benzene; $\Delta$ and --.---, chloroform; $\bullet$ and -_-, 1-octanol; and $\square$ and -_-_-_, 2octanone, respectively.

the mechanism of the solvent effects on the dimerization of $\mathrm{CuA}_{2}$ in the organic phase has proven to be distinct from that on the partition of $\mathrm{CuA}_{2}$ between the aqueous and mixed-solvent phases. On the other hand, in the 1octanol-benzene mixed-solvent systems the plots of No. 11 and 12, which were shifted to the left from the straight line in Fig. 1, fit a straight line with a slope of -2 , as shown in Fig. 2. This suggests that both $K_{\mathrm{D}, \mathrm{CuA} 2}$ and $K_{2, \mathrm{CuA} 2}$, which are plotted in Fig. 2, are mainly influenced by the solvation of the extracted species $\left(\mathrm{CuA}_{2}\right)$ by a 1octanol molecule.

Comparison of mixed solvents with benzene, 1-octanol, 2octanone and chloroform on the extractability of copper(II) benzoates

The extraction curves for the mixed solvents No. 6 and 18 drawn on the basis of the results obtained by the present study are shown in Fig. 3 together with those for benzene, 1-octanol, 2-octanone and chloroform based on a previous study. ${ }^{1}$ The observed values fit well in the calculated extraction curves, and the validity of the present results are supported. The mixed solvents No. 6 and 18 containing $1.0 \mathrm{~mol} \mathrm{dm}^{-3} \quad 1$-octanol and $1.0 \mathrm{~mol} \mathrm{dm}^{-3}$ 2-octanone, respectively, in benzene were found to be most useful in the respective mixed solvents for the extraction of copper(II) benzoates. In Fig. 3, these mixed solvents (No. 6 and 18) are shown to be slightly superior to chloroform in the extraction of copper(II) benzoates.

As shown in Tables 1 and 2 , the extractability of copper(II) benzoates varies with the mixing ratios of two kinds of solvents. For 1-octanol-benzene and 2octanone-benzene mixed-solvent systems, the respective representative extraction curves were drawn on the basis of the present results given in Figs. 4 and 5, respectively. 


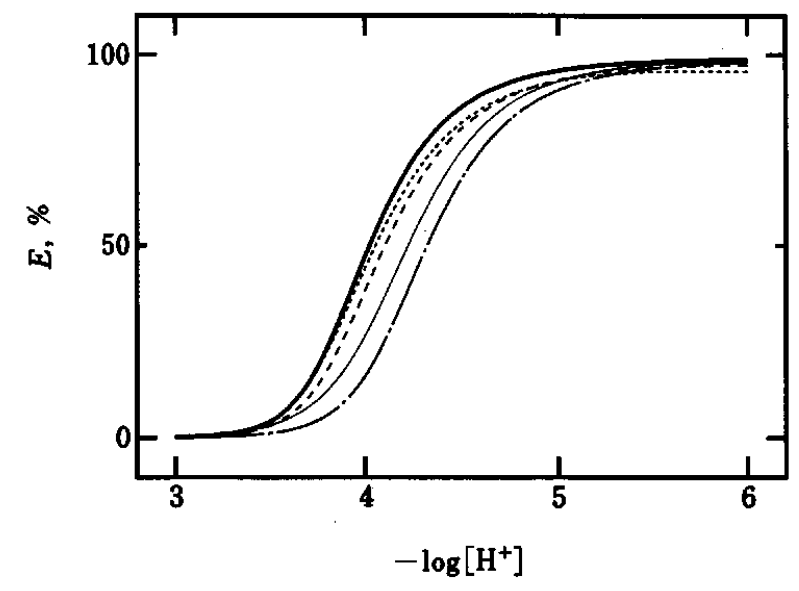

Fig. 4 Extraction curves in the extraction systems using the 1octanol-benzene mixed solvents. The lines are as follows: ----, mixed solvent No. 1; - mixed solvent No. 6; --_-_, mixed solvent No. 8; and - - , mixed solvent No. 11 , respectively.

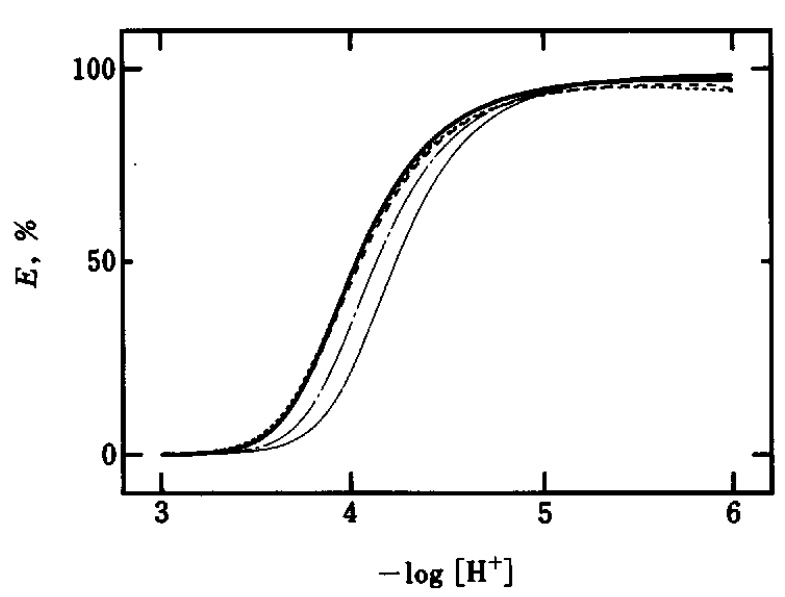

Fig. 5 Extraction curves in extraction systems using the 2octanone-benzene mixed solvents. The lines are as follows: - , mixed solvent No. 13 ; - -, , mixed solvent No. 15 ; mixed solvent No. 18; - -_._-, mixed solvent No. 20; and - , mixed solvent No. 23 , respectively.

No. 6 and 18, that are shown in Fig. 3, were drawn again for a comparison.

The percent extraction closely correlates with the various equilibrium constants listed in Tables 1-3; that is, the extractability is fixed by a balance of these equilibrium constants. Judging from the results obtained by the present work, mixed solvents No. 2, 3 and 5 were found, as well as No. 6 and 18, to be slightly superior to chloroform under the present extraction conditions (at $C_{\mathrm{HA}}=0.5, C_{\mathrm{Cu}, \mathrm{tot}}=5 \times 10^{-3} \mathrm{~mol} \mathrm{dm}^{-3}$, and at $\mathrm{pH}=3,4,5$ and 6 ). In the extraction system with mixedsolvent No. 4 the dimerization constant of $\mathrm{CuA}_{2}$ in the organic phase is smaller than that in systems with mixed solvents No. 3,5 and 6. This is the reason that the former solvent is inferior to chloroform in the extraction of copper(II) benzoates in contrast to the latter solvents. The larger values of $K_{\text {ex(10), }}, K_{\text {ex(20) }}$ and $K_{\text {ex(21) }}$ in the No. 2 mixed solvent than the values in the No. 4 cause the better extractability of copper(II) benzoates than the latter solvents. In the case of chloroform, the smaller value of $K_{\text {1).IIA }}$ than the latter solvent can be expected to be one of the causes for lowering the extractability than that in No. 2 system.

In those extraction systems with the 2-octanonebenzene mixed solvents, only No. 18 is superior to chloroform in the extraction of copper(II) benzoates. In these mixed-solvent systems although the dimerization constant of $\mathrm{CuA}_{2}$ in the organic phase is considerably larger than that in the 1-octanol-benzene mixed-solvent systems, the partition constant of $\mathrm{CuA}_{2}$ is fairly smaller than that in the systems shown in Table 3. This lower partition constant of $\mathrm{CuA}_{2}$ may cause the extractability in the former mixed-solvent systems to be inferior to that in the latter mixed-solvent systems.

As mentioned above, in the present study the mixed solvents containing a certain content of 1-octanol or 2octanone in benzene were found to be superior to not only benzene, 1-octanol and 2-octanone, but also chloroform for the extraction of copper(II) benzoates. This result can be regarded as being a synergistic effect in a sense. These useful effects can be expected to result from a change in the property of the solvent as a whole by mixing solvents with different capabilities and the occurrence of some solvating ability by adding a solvating solvent to a nonsolvating solvent.

Consequently, such mixed solvents as the present ones will be effective for the solvent extraction of metal ions and will serve in place of useful halogenated solvents, such as chloroform and 1,2-dichloroethane, the use of which is now voluntarily controlled. Benzene is also known to be carcinogenic. But, in the present study, we used it as a component of the mixed solvents in order to compare with the results previously obtained with neat benzene. Some saturated hydrocarbons, such as octane, can be expected to be useful in place of benzene as a non-solvating component of the mixed solvents.

The present work was supported by a Grant-in-Aid for Scientific Research (No. 06640780) from the Ministry of Education, Science, Sports and Calture.

\section{References}

1. H. Yamada, K. Yajima and H. Wada, Anal. Sci, 11, 715 (1995).

2. H. Yamada, Y. Taguchi and H. Wada, Talanta, 41, 573 (1994).

3. H. Yamada, K. Yajima, H. Wada and G. Nakagawa, Talanta, 42, 789 (1995).

4. H. Yamada, K. Takahashi, Y. Fujii and M. Mizuta, Bull. Chem. Soc. Jpn., 57, 2847 (1984).

5. H. Yamada, S. Horikawa, Y. Fuiii and M. Mizuta, Bull. 
Chem. Soc. Jpn., 61, 835 (1988).

6. H. Yamada, R. Kitazaki and I. Kakimi, Bull. Chem. Soc. Jpn., 56, 3302 (1983).

7. M. Tanaka, Solvent Extr. Proc. Int. Solvent Extr. Conf., p. 16 (1971).

8. Y. Fujii, K. Sobue and M. Tanaka, J. Chem. Soc. Faraday
Trans. I., 74, 1467 (1978).

9. S. Oki, T. Omori, T. Wakahayashi and N. Suzuki, $J$. Inorg. Nucl. Chem., 27, 1141 (1965).

(Received January 25, 1996) (Accepted March 29, 1996) 